\title{
Molecularly Imprinted Polymers as Selective Receptors for Sensing Nanosized Species
}

\author{
Suticha Chunta ${ }^{1}$, Christoph Jungmann ${ }^{1}$, Leo Schranzhofer ${ }^{1}$, Roongnapa Suedee ${ }^{2}, \underline{\text { Peter A. Lieberzeit }}{ }^{1}$ \\ ${ }^{1}$ University of Vienna, Faculty for Chemistry, Department of Physical Chemistry, Vienna, Austria \\ ${ }^{2}$ Department of Pharmaceutical Chemistry, Prince of Songkla University, Hatyai, Thailand \\ Peter.Lieberzeit@univie.ac.at
}

\begin{abstract}
Molecular imprinting into highly cross-linked polymers has attracted substantial interest for a wide range of analytes. Herein, we present two application examples of molecularly imprinted polymers (MIP) as artificial receptors for chemosensors: both analytes, namely high densitiy lipoprotein (HDL) and engineered gold nanoparticles, respectively, share their dimensions in the range of a few ten $\mathrm{nm}$. Utilizing the resulting surface MIP as artificial receptors on quartz crystal microbalance (QCM) sensors leads to appreciable results. In both cases, MIP yield much higher sensor responses (up to an order of magnitude higher), than their non-imprinted counterparts. MIP sensors reveal dynamic sensor signals, for HDL in the physiologically interesting range. The two examples hence show the potential of molecular imprinting for designing receptor layers targeting analytes in the nanometer range.
\end{abstract}

Key words: Molecular imprinting, mass-sensitive sensors, biomimetic recognition, nanoparticle sensors, lipoprotein sensor.

\section{Introduction}

Molecularly imprinted polymers attract increasing scientific interest and have found applications in such diverse areas as preconcentration, separation, chemical sensing, catalysis, and others [1]. The rationale behind their synthesis is outlined in Figure 1:
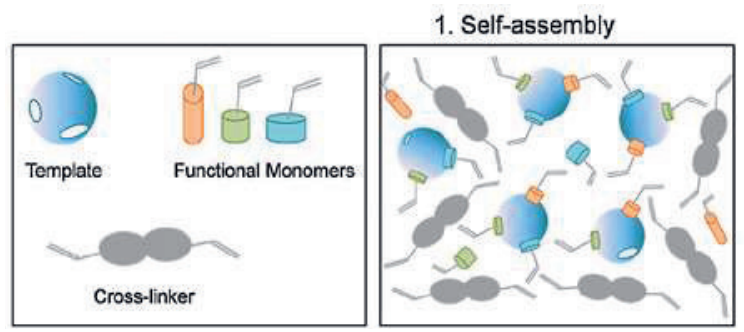

2. Polymerisation

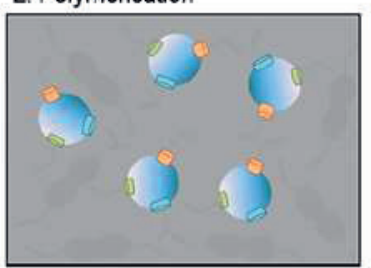

3. Removal of Template

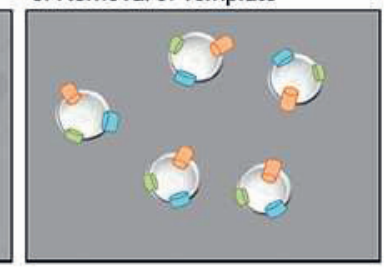

Fig. 1. Principle of molecular imprinting. Reproduced with permission from [2]. @ Elsevier B.V.

Basically, the approach comprises polymerizing a highly cross-linked matrix in the presence of a template component. Pre-organization between this template and (functional) monomers prede- fines a non-covalent interaction network between functional groups of the polymer and the template. After hardening and removing said template, cavities remain in the matrix. They exactly fit the steric and functional properties of the respective template and are hence useful for re-incorporating those compounds in a selective manner.

Such recognition behavior has made MIP very interesting for the design of novel, highly selective chemical sensors, because they combine bio-analogous recognition abilities with the ruggedness and processability of manmade polymers. Hence, a wide variety of MIP sensor applications has been suggested so far, with analytes ranging from small molecules [2], to viruses [3] and bacteria [4]. Among these classes, nanometer-sized species deserve special attention, because to date hardly any rapid analysis techniques exist in this size range: Those species are too small to be accessible by light scattering techniques and usually also cannot be assessed by impedance measurements/impedance spectroscopy.

Within this paper we present two MIP strategies for such nanosized species, namely high density lipoprotein (HDL) and engineered nanoparticles. Whereas the former are potentially highly interesting for diagnostic purposes, the latter topic is of more general interest, especially in 
the fields of environmental monitoring and food safety.

\section{High density lipoprotein MIP}

$\mathrm{HDL}$ is one of several classes of cholesterolbased lipoproteins. Due to its involvement in atherosclerosis it serves, amongst others, as an important biomarker. It consists of a composite of cholesterol, lipids and proteins. In the blood stream, it is present as a globular structure with roughly $10 \mathrm{~nm}$ in radius [5]. While a sensor has already been reported for low density lipoprotein (LDL) [6] no comparable system exists for HDL. The reason most probably can be found in one of the main analytical challenges in this case: the concentration of $\mathrm{HDL}$ in physiological/clinical samples is usually lower (ideally $>60 \mathrm{mg} / \mathrm{dl}$, in clinical cases down to below $15 \mathrm{mg} / \mathrm{dl}$ compared to 25 to $>200 \mathrm{mg} / \mathrm{dl}$ for LDL). Nonetheless, the polymers used to synthesize LDL MIP turned out suitable starting points for designing HDL MIP sensors. Again, a copolymer of methacrylic acid (MAA) and vinyl pyrrolidone (NVP) cross-linked with ethylene glycol dimethacrylate (EGDMA) as the cross linker turned out most useful. The optimized matrix brought sensitivity of respective MIPcoated quartz crystal microbalance (QCM) transducers down to the clinically relevant range. Such sensors were able to detect HDL concentrations well below $100 \mathrm{mg} / \mathrm{dL}$. Sensor signals for samples containing $50 \mathrm{mg} / \mathrm{dl}$ and $12.5 \mathrm{mg} / \mathrm{dl}$ (see Fig. 2), respectively, are well above the noise level of the measurement, which in this case is in the range of a few $\mathrm{Hz}$. The NIP shows anti-Sauerbrey behavior, which is to be observerd frequently with globular particles on flat surfaces. The beauty of the approach lies in the fact that in contrast to cur-

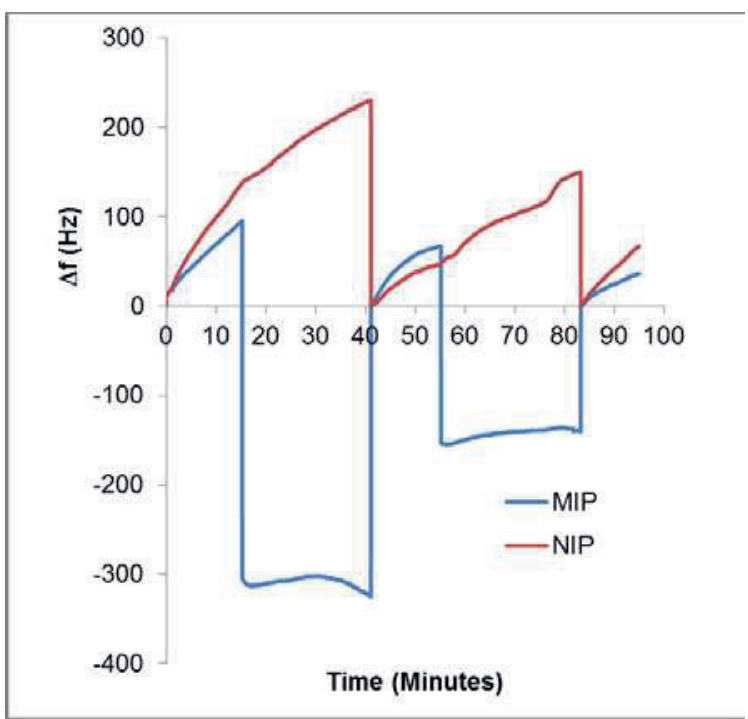

Fig. 2. Sensor QCM sensor responses of HDL-MIP and respective NIP toward to HDL standards in PBS buffer $(\mathrm{pH}=7.4)$ rent clinical tests, the sensor proposed here detects HDL itself rather than assessing its amount via the $\mathrm{HDL}(\mathrm{C})$, i.e. cholesterol content. This is also interesting from the MIP point of view, because hardly any imprinting has been reported on biochemical aggregates so far.

\section{MIP for Engineered Nanoparticles}

Nanoparticles are defined as objects of uniform size and shape in the region of $1-100 \mathrm{~nm}$ and have attracted steadily increasing attention during the last decade: due to their size, their physical properties (reactivity, surface energy etc.) more closely resemble those of surface atoms than those of bulk atoms. This leads to promising technological applications, among others in the fields of cosmetics/personal healthcare and food industry, respectively. However, engineered nanoparticles have come under close scrutiny as a result of this in an ever increasing number of commercial products: Even materials which are otherwise known to be harmless may pose a risk to public health when scaled down to nanometer size. This can be explained by the inherently high surface-to-volume ratio of nanoparticles and their small size which allows them to pass through cell membranes. Although this is known, only few data exist on toxicology of nanoparticles and their pharmacokinetics. Same can be said about the effects resulting from human long-term exposure to NPs This makes analyzing them a pressing issue.

In the course of the research work shown three different methods were developed and optimized for nanoparticle imprinting. These include: one-step, two-step and sedimentation imprinting. Based on affinity tests carried out on different polymer systems it was found that polyurethane yielded the most appreciable

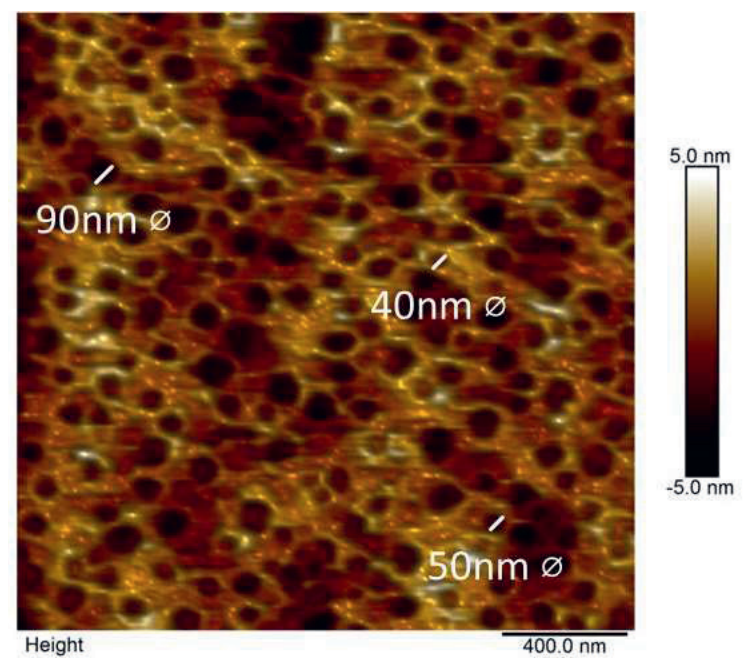

Fig. 3. AFM image of Au NP-MIP surface 
results with sensor effects generally being reversible as well as reproducible. First results of these experiments were published for $\mathrm{Ag}$ nanoparticles [7]. The concept, however, is not limited to silver nanoparticles as laid out there. As can be seen in Figure 3, gold nanoparticles also lead to very appreciable imprinted cavities in the polymer surface. This is appreciable, yet not too surprising, because diameters of these engineered nanoparticles are in the same size range, as those of viruses. Actually, several MIP have already been published for those (some recent examples can e.g. be found in $[3,8,9])$. Figure 4 shows QCM results revealing that gold NP also lead to appreciable sensor

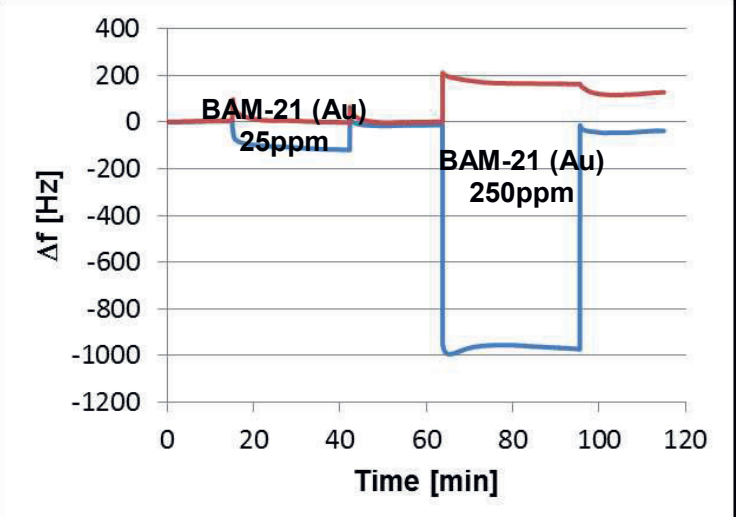

Fig. 4. QCM sensor responses of MIP (blue) and NIP (red), respectively, toward Au NP.

responses. Obviously, the mass sensitive signals depend on nanoparticle concentration and can be traced back to molecular imprinting: signals on the electrode coated with the nonimprinted material (non-imprinted polymer NIP) lead to sensor responses that reach only about $25 \%$ of the corresponding MIP. Given the high density of gold $\left(\rho=19.2 \mathrm{~g} / \mathrm{cm}^{3}\right)$ these are very appreciable results. Nonetheless, a range of questions still wait to be answered, including e.g. the influence of the respective stabilizer shell on the sensor responses caused by a nanoparticle. Furthermore, selectivity of the systems also requires further experiments.

\section{Summary and conclusion}

The two analytes presented herein are from very different origin: HDL is obviously a natural compound that is present in the human blood stream; engineered nanoparticles (PVPstabilized gold in the concrete case) on the other hand represent fully artificial species. However, they have their physical dimensions in common (in the concrete case, both species have a radius just below $10 \mathrm{~nm}$ ). In both cases it is possible to generate MIP, which demonstrates the generality of the synthetic approach and closes an "analytical gap" between sensors for small molecules and those aiming at detecting larger species, such as bacteria and entire cells. Despite appreciable results, several points remain a challenge, for instance the fact that surface roughness of MIP is also in the nm-range. Despite such current shortcomings, both HDL and NP sensors seem fit for use in real-life matrices. The former ones lead to appreciable sensor responses near the physiologically interesting concentration range.

\section{Acknowledgements}

The work on NP MIP was funded by the European Commission within the seventh research framework EU-FP7, project number EU-FP7-NMP-2011-SME-5-NMP4-SE-2012280550 „INSTANT“, which we gratefully acknowledge. CS gratefully acknowledges support of her PhD studies by the Royal Thai Government through a scholarship granted by the Office of the Higher Education Commission (Grant No. 04/2556), Thailand. We also gratefully acknowledge the Faculty of Medical Technology and and the Faculty of Pharmacy, Prince of Songkla University for blood collection and ultracentrifugation.

\section{References}

[1] B. T. S. Bui, K. Haupt, Analytical and Bioanalytical Chemistry 398, 2481-2492 (2010); doi: $10.1007 / \mathrm{s} 00216-010-4158-x$

[2] J. Wackerlig, P. Lieberzeit, Sensors and Actuators B: Chemical 207, 144-157 (2015); doi: 10.1016/j.snb.2014.09.094

[3] T. Wangchareansak, A. Thitithanyanont, D. Chuakheaw, M. Gleeson, P. Lieberzeit, C. Sangma, MedChemComm, 617-621 (2014); doi: 10.1039/c3tb00027c

[4] R. Samardzic, H. Sussitz, N. Jongkon, P Lieberzeit, Sensor Letters 12 (6-7), 1152-1155 (2014); doi: 10.1166/sl.2014.3201

[5] T. Arai, T. Tsukada, T. Murase, K. Matsumoto, Clinica Chimica Acta 301, 103-117 (2000); DOI: 10.1016/S0009-8981(00)00328-4

[6] S. Chunta, R. Suedee, P. A. Lieberzeit, Analytical Chemistry 88, 1419-1425 (2016); DOI: 10.1021/acs.analchem.5b04091

[7] P. A. Lieberzeit, C. Jungmann, L. Schranzhofer, Procedia Engineering 87, 236-239 (2014); DOI 10.1016/j.proeng.2014.11.630

[8] Z. Altintas, J. Pocock, K.-A. Thompson, I. E. Tothill, Biosensors and Bioelectronics 74, 9961004 (2014); DOI: 10.1016/j.bios.2015.07.076

[9] J. Zhou, N. Gan, T. Li, F. Hu, X. Li, L. Wang, L. Zheng, Biosensors and Bioelectronics 54, 199206 (2014); DOI: 10.1016/j.bios.2013.10.044 